heils war, durch starke Blutcongestionen nach dem Kopfe waren $z$ war die sensorielten Functionen nicht frei geblieben, aber die Störung derselben nicht ron grofser Bedeatung ; was den Zussand am gefährlichsten machto, war ein Asthma. welches Ersticliung zu drohen schien.

Das Sirychnin wurde sogleich entfernt; die wunde Stelle war weder entzündeter noch einpfindlicher, als dies bei Ve. sicatorien der Fall an seyn pllegt. Zunächst wurden ableitende hiystiere vernidnet, und zum innern Gebranch verdünnte, Essig, da nıa wegen der Congestionszufälle nicht wagen morhte Opium zu reichen. Nach einigen Stunden nahmen zwar die Erscheinungen ab, aber Schlaflosigkeit und Adynamie des Hörpers dauerten mehrere Tige. Das gelahnt: Glied aber zeigte eine ouffallend vermehte Starlte und Bratchbarleit. Nach 8 Tagen wurde die Anwendung des Stryclmins aaf nbiga. Weise wiederholt und dieselben Resultate prhalten, und dann ferner in Zwischenräumea ron 8-10 Tagen damit fortgetahren. Die getihhlichen Wirkungen des Mitiels tratun bei jeder Wiederholuar milues aut, wie Heilun: aber scbritt so sicher fort, dal's nach zehminaliger Inwendung die Brante wieder stricken, dam nähen und sticken und sein leicht schreiben liounte.

\title{
Arzneiliche Zubereitungen von Schnecken You
}

Emil Mourihon, Apocheker z u Lyor.

(Auszug aus dem Journal de Phajmacie, XIX. 183.)

Es scheint mix am angemessensten, die Schnecten in einem gewissermafsen vitalen Zustande anzuwenden, nach Art der Austern, um das icucilaginöse Princip derselben, worin ihre Annal.d Pharin. VI, Bds, 3. Heft. 


\section{4}

Wirksamkeit besteht, möylichst unzersetzt zu erhalten, weil sie in solchern Zastande als erweichendes und als Brust-Mittel am heilsamsten wixken.

Es ist nicht einzusehen, aus welchem Grunde man der Helix pomatiu für den medicinischen Gebrauch ausschliefslich den Vorzug griebt Helic vermicularis, H. rhodostonia und H. nemoralis und im Aligemeinen alle Schnecken, welohe zu den grofsen Arten gehören, sind eben so gut. Wollte man einen Unterschied dazwischen machen, so könnte dieses nur vielleicht insofern seyn, als sie als Nahrungsnittel dienen.

Es ist bchannt, dâfs man die Sohnecken durch ein mehr oder weniger langes Fasten von ihrem unangenehmen Geschmach, wie von den schädlichen Eigenschalten, welche einige durch zufällige von ihrer besondern Nahrung abhängige Modificationen erlitten haben können, befreiet. Hierdurch aber verlieren sie den grölsten Theil ihrer schleimigten Materie, und äberhaupt hat man selten nöthig dieses Vorfahren zu beobachten.

Sclınecken-Zucker, Sucre hèliciè, Saccharum heliciàtum.

Schnecken ron einer guten Art, 256 Stüch, die ohne Gebäı:se und Eingeweide wiegen 45 Unzen Zucker Wasser. . . . . . . . . . . . . 128

Das Fleisch der gereinigten und zerscbnittenen Schnechen wird eine Viertelstunde lang $\mathrm{mtt}$.. $\mathrm{nn}$ Wasser stark gescblagen, ausgedrüclit und danr mit dern Zucher unter stetem Umrührea rasch zur l'rockne verdampft. Das Produkl wiegt 128 Unzen. Es schmeckt angenehm; mufs in hermetisch verschlossenen Gläsern aufbewahrt werden. Man begreift leicht, wie man ron diesem Saccharolat zu jeder Jahreszeit Gebrauch machen kann. 


\section{Schnecken - Zeltchen.}

Schnecken-Zucker

16 Uizen

Traganth

1 Drachme

Orangenblüthenwasser .. $11 / 2$ Unzen

Man macht davon Zeltchen, das Stüch 2416 Gran.

$$
\text { Schnccken - Mucilago. }
$$

Schnecken . . . . . Nro. 4.

Zuckersyrup .. . . . 6 Drachmen

Orangenblüthenwasser . . 2 -

Brunnenwasser . . . . 3 Linzen

Die vom Gehäuse und den Eingeweiden befreiten Schnechen werden mit den Wasser zu Mucilago gemacht, nachber die übrigen Theile zugesetzt. Die Vorschrift ist fïr eine Dosis, die des Morgens genommen wird. Auch hann man diese mit mehr Zuckerwasser rertünnen und so als tägliches Getränk für den Kranten verwenden.

Schnecken-Gelée, Gelatina heliciata, Gclée hèlicié.

Grofse Schnecken . . . Nro. 4.

Hausenblase . . . . . , Drachme

Zuckersyrup . . . . . , Unze

Wasser . . . . . . . 3 Unzen

Mit 2 Unzen Wasser und den gereinigten Sclunecken macht man ein Mucilago, löst die Hausenblase in der übri. gen Flüssigheit auf, mischt alles zusammen, lärst es durch ein Tuch laufen und setzt etwas Citronenolzucker za. An einem küblen Orte gesteht die Masse bald zu einem durchscheinenden Gelée, und kann sich auch bei +10 bis $12^{\circ} \mathrm{n}$ mehrere Tage halten.

$$
\text { Schnecken - Syrup. }
$$

Präparirte Schneclien Nr. $128 \quad 24$ Unzen

Tuckersyrup . . . . . 128 -

Brunnenwasser . . . . 64 -

Orangenblüthenwasser . . . 3 - 
Man läst deı Zuckersyrup einkochen bis zum Gewicht der aus den Schnecken mit dem $\mathbf{W}_{\text {asser }}$ bereiteten Mucilago, giebt letztere zu dem lochenden Saft, gielst durch und setzt. dann das Orangenblüthenwasser zu.

$$
\text { Sohnecken - Paste. }
$$

Treganth

llausenblaseu

Grofse Schnecken Nr. 6if, ohne Ge-

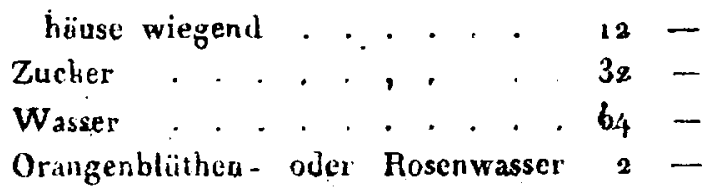

Mit dem Traganth und 28 Unzen Wasser wird in der Kälte eine Murilago beteitet, in 15 Unzen derselben lüst mao in der Wärme die Hausenblase anf', gereinigt heide Flüssigkeiten und driickt sie durch ein Tuch. Mit den übrigen 32 Unzen verden die Schnecken behandelt, geschlagen und die Flïssigleit ehenfalls durch Leinewand sedrückt.

$\Lambda$ ul mäsigen Feuer wird nun die erste gummihaltige Flüssigkeit unter stetem Agitiren bis zur Consistenz eines dicken Honigs gebracht und nun in Kleinen Portionen der zu Schsum geschlagene Schneckenschlein zugemischt. Wenn die Paste die gehörige Cunsistenz bat, setzt man das aromatische Wasser hinzu, und bringt sie dann auf eine mit Stärkmehl bestreuete Marmorplatte. Sie hat fast das Ansehen dor Althäfasta, ist weniger compact und mufs gegen cine teuchte Atinosphäre geschiitzt aufbewahrt werden. Wenn sie dẹn zu ihrer Conscrvation nöthigen Grad von Kochung erlitten hat, darf sie nur \& Pfund wiegen.

Diese sämmtlicheu Präparate, mit Ausnahme des Syrups, melhalten in einer. Unze das Product ron zwei Schnecken.

Heines dieser Vedicamente, selbst die Mucilago und Gelet, wenu dazu Scliwecken gebraucht wurden, die einige Tage 
einern Fasten unterworfen waren, bietel das geringste Unangenebrie dar.

\section{Der Xereswein.}

Der Wein vom Xeres gehört belanntlich zu dell rorzüglicheren. Sorten der feurigen spanischen Weine und wir theilen darüber nachfolgende Pemerkungen mit, im Auszuge aus den Raisen der Heren Inglis and Brooke in Spanien und Maroceo.

Die Weingärten von Xeres lïunsen einen Raum von 6 engl. Quadratmeilen bedecken; sie liegen aler nisht neben einander sondern zerstreut, aul abschüssigen Hügeln. Man zieht die Stöcke dicht am Borlen hin, damit der Wein dadurch mehr Hitze erhầlte.

Die rechte Iraube für den Xeres ist grün; erst veun sie völlig reil und anfängt einzuschrumpfen, wird sie gepflickt. In Jahren mittler Güte fängt tiu Ernte am gtets, ia schlechtern Jahrgängen erst am sóten scptember, nie aber spater an.

Der. Boden der Weingärten un Xeres ist reicher als in St. lucar, Muerto Reale und St. Maria In diesen Orten fängt die Weinlese früher an als in Xeres. Die Weine sind aber bleicher und haben weniger Hörper und Blume als Xeres. Sie sind die geringeren Gattungen Xeres, bethinnt unter den Namen Manzanilla und St. Lucur.

Bei sorgfältiger Lese häufen die Winze: die geptlückten Trauben in Körben auf, und lassen sie, unter öfterem Umweuden, 48 Stunden der Luft und Sonne ans fesetzt. Sie erhalten nun zwar eine geringere Menge Saft, aber einen bessern Wein.

Das Pressen der Trauben geschieht auf folgende Weise. Drei bis vier Männer treten dieselben mit mit Nägeln iseschla- 